\title{
Nrf2 Alleviates TNF-a-Induced Oxidative Stress Damage in Type II Alveolar Epithelial Cells by Down- Regulating the Expression of NOX1
}

\section{Weijing Wu}

Second Affiliated Hospital of Fujian Medical University

Jiamin Zhang

Fujian Medical University Affiliated First Quanzhou Hospital

Xihua Lian

Second Affiliated Hospital of Fujian Medical University

Xiaoping Lin

Second Affiliated Hospital of Fujian Medical University

Xiaoshan Su

Second Affiliated Hospital of Fujian Medical University

Li Zhu

Second Affiliated Hospital of Fujian Medical University

\section{Zesen Zhuang}

Second Affiliated Hospital of Fujian Medical University

\section{Zhixing Zhu}

Second Affiliated Hospital of Fujian Medical University

Yiming Zeng ( $\nabla$ ymzeng08@163.com )

Second Affiliated Hospital of Fujian Medical University https://orcid.org/0000-0001-6429-0682

\section{Research}

Keywords: Nrf2, NOX1, TNF-a, acute lung injury, oxidative stress

Posted Date: September 3rd, 2020

DOl: https://doi.org/10.21203/rs.3.rs-68779/v1

License: (c) (i) This work is licensed under a Creative Commons Attribution 4.0 International License.

Read Full License 


\section{Abstract}

Objective: To study the roles of Nrf2 in acute lung injury (ALI) pathogenesis by investigating the effects of Nrf2 on regulating oxidative stress damage in TNF-a-induced type II alveolar epithelial cells $\left(\mathrm{T}_{2} \mathrm{AECs}\right)$.

Methods: $T_{2} A E C s$ were transfected with Nrf2 siRNA and overexpression vectors for six hours before being induced by TNF-a for 24 hours. Subsequently, levels of interleukins (IL-6 and IL-8), reactive oxygen species (ROS), malondialdehyde (MDA), total antioxidation capability (T-AOC), Nrf2, NOX1 and NF-kB were measured. Additionally, potential Nrf2 binding site in NOX1 promoter was predicted by AliBaba2.1 and two recombinant vectors, namely "pGL3-NOX1-1500" and "pGL3-NOX1-1489, were constructed by inserting the sequence of NOX1 promoter in full-length and that in the absence of Nrf2 binding site to pGL3 basic vector. $T_{2} A E C s$ were transfected with these vectors prior to TNF-a induction and the luciferase activity was measured.

Results: Levels of IL-6, IL-8, ROS and MDA were increased ( $P \otimes 0.05)$ while T-AOC was decreased in TNF-ainduced A549 cells after the transfection of Nrf2 siRNA vector (P凶0.05). In contrast, concentrations of IL-6, IL-8, ROS and MDA were decreased ( $P \otimes 0.05)$ whereas T-AOC was increased after the transfection of Nrf2 overexpression vector $(P \otimes 0.05)$. NOX1 promoter possesses one Nrf2 binding site. Cells transfected by "pGL3-NOX1-1500" vector had the highest luciferase activity, followed by cells transfected by "pGL3NOX1-1489" vector and the control cells (Pख0.05).

Conclusion: Nrf2 modulates NOX1 expression via binding to its promoter, by which against TNF-ainduced oxidative stress damage in $\mathrm{T}_{2} \mathrm{AECs}$. Thus, Nrf2 might be a therapeutic target for ALI.

\section{Introduction}

Acute lung injury (ALI) is a common and lethal complication of pulmonary and extrapulmonary diseases, of which the overall mortality rate is extremely high, approximating to $40 \%{ }^{[1-3]}$. Moreover, ALI has been treated as the primary death cause of numerous illnesses, and it has become the main source of mortality in COVID-19 ${ }^{[3,4]}$. However, the pathogenesis of ALI remains yet clear so far. Diffused alveolar damage is the predominant pathological feature of $\mathrm{ALI}{ }^{[5]}$. Moreover, hyperactive inflammatory reaction and excessive oxidative stress are the major mechanisms by which type II alveolar epithelial cell ( $\left.\mathrm{T}_{2} \mathrm{AECs}\right)$ are extensively injured under $\mathrm{ALI}^{[6]}$. Thus, maintain the inflammatory response and oxidative stress to their appropriate levels in $T_{2} A E C s$ is crucial for ALI therapy.

Imbalanced oxidative stress is mainly caused by dysregulated generation of reactive oxygen species (ROS) and excessive consumption of antioxidants ${ }^{[7]}$. NOX family members are the master contributors of ROS, and the production of ROS in lungs is mainly maintained by NOX1 ${ }^{[8]}$. Moreover, aberrant NOX1expression in $\mathrm{T}_{2} \mathrm{AEC}$ r results in excessive oxidative stress, contributing to the pathogenesis and development of ALI ${ }^{[9]}$. Therefore, reduce NOX1-mediated ROS production might be able to effectively alleviate the inflammation reaction and oxidative stress damage in $\mathrm{T}_{2} \mathrm{AECs}$ under ALI. Nrf2 is an 
important transcription factor and plays crucial roles in regulating the transcription activity of a wide range of antioxidant genes ${ }^{[10,11]}$. Generally, Nrf2 is ubiquitinated and degraded because of the mediation of Keap1, however, under oxidative stress challenge, the Nrf2-Keap1 complex is dissociated, which allows Nrf2 to translocate into the nucleus, resulting in the activation of its downstream genes, by which it exerts its role in modulating the levels of oxidative stress, inflammation, apoptosis, and autophagy ${ }^{[10]}$. To date, with the roles of Nrf2 in preventing the occurrence or mitigating the severity of ALI or acute respiratory distress syndrome after it was activated were discovered in a myriad of studies, the significance of Nrf2 in terms of protection against ALI have been firmly demonstrated ${ }^{[12,13]}$.

TNF-a is not only a pivotal proinflammatory cytokine, playing a crucial role in the trigger of inflammatory reaction and oxidative stress, but it also is a robust ALI pathogenic factor because of its central roles in the induction of $\mathrm{ALI}^{[14,15]}$. Hence, TNF-a has been widely used to establish ALI in vitro model ${ }^{[16]}$. Previously, we found that the expression of Nrf2 and NOX1 were increased simultaneously in TNF-ainduced A549 cells, and recently, a potential Nrf2 binding site in NOX1 promoter was predicted using AliBaba2.1 database, indicating that the interaction between Nrf2 and NOX1 might play significant roles in the pathogenesis and progress of ALI. Nonetheless, whether Nrf2 can regulate the expression profiles of NOX1, subsequently leads to the changes of inflammation reaction and oxidative stress damage in $\mathrm{T}_{2} \mathrm{AEC}$ s induced by TNF- $\mathrm{a}$ is still unclear. Therefore, in this in vitro study, TNF-a-induced A549 cells were used, and the effects of Nrf2 on regulating the levels of interleukins (IL-6 and IL-8), ROS, malondialdehyde (MDA), total antioxidation capability (T-AOC), NOX1 and NF-kB were measured. In addition, luciferase activity was determined to further investigate the underlying mechanism by which Nrf2 can modulate the expression of NOX1.

\section{Materials And Methods}

\section{Reagents}

Roswell Park Memorial Institute 1640 Medium, 0.25\% trypsin-EDTA, and FBS were purchased from HyClone (Logan, USA.). Nrf2 siRNA vector and Nrf2 high expression vector were designed and synthesized by RiboBio Company (Guangzhou, China) and Gene Chemical Technology (Shanghai, China). Recombinant vector "pGL3-NOX1-1500" and "pGL3-NOX1-1489" were synthesized by GenePharma (Suzhou, China). Lipofectamine ${ }^{\mathrm{TM}} 2000$ was bought from Invitrogen (California, USA.). PrimeScriptTM RT (RR037A) and SYBRR premix Ex TaqTM (RR420A) reagent kits were purchased from TaKaRa (Osaka, Japan). Primers of human GAPDH, Nrf2, NOX1, and NF-kB were ordered from Sangon Biotech (Shanghai, China). Primary monoclonal antibodies of human GAPDH (ab181602), Nrf2 (ab62352), and NOX1 (ab78016) were purchased from Abcam (Cambridge, UK) and NF-KB p65 (8242S) was purchased from Cell Signaling Technology (Massachusetts, USA). Goat anti-rabbit horseradish peroxidase-conjugated secondary antibody was bought from Beyotime (Shanghai, China). TNF-a was purchased from PeproTech (New Jersey, USA). IL-6 and IL-8 ELISA kits were bought from R\&D (Minnesota, USA). Reactive 
Oxygen Species Kit, Lipid Peroxidation MDA Kit, and Total Antioxidant Capacity Assay Kit were ordered from Beyotime (Shanghai, China).

\section{Cell culture}

A549 cells were cultured in RPMI-1640 medium with 10\% FBS and kept in an environment containing 5\% $\mathrm{CO}_{2}$ at $37^{\circ} \mathrm{C}$. They were passaged every three days and cells used for experiments were incubated in sixwell plates. According to different purposes, cells were randomly divided into three parts. Specifically, cells in first part were randomly assigned into the control group and TNF-a treated group; those in second part were randomly divided into naive group, TNF-a-treated group, negative control group, Nrf2 siRNA group, Nrf2 overexpression group, and empty vector group; and those in third part were randomly divided into pGL3 group, pGL3-NOX1-1500 group and pGL3-NOX1-1489 group.

\section{Cell treatments}

Cells in control group were cultured in normal medium while cells in TNF-a-treated group were cultured with $2.5,5,10,20$ and $40 \mathrm{ng} / \mathrm{ml}$ TNF-a for 24 hours or cultured with $10 \mathrm{ng} / \mathrm{ml}$ TNF-a for $0,6,12,24,36$ and 48 hours, respectively.

Cells in naive group were cultured in normal medium, while cells in TNF-a-treated group were stimulated by $10 \mathrm{ng} / \mathrm{ml}$ TNF-a for 24 hours. Vector transfection was performed using lipofectamineTM 2000. Cells in the remaining groups were transfected with negative siRNA vector ( $50 \mathrm{nmol} / \mathrm{L})$, Nrf2 siRNA vector (50nmol/L), Nrf2 high expression vector (4ug), and empty vector (4ug) for six hours, followed by stimulation of $10 \mathrm{ng} / \mathrm{ml}$ TNF-a for 24 hours.

Cells in third part were transfected with pGL3 basic plasmid, and those in pGL3-NOX1-1500 plasmid and pGL3-NOX1-1489 plasmid, respectively.

\section{ELISA assay}

Cell supernatants in each group were collected and levels of IL- 6 and IL-8 were measured by ELISA assay in accordance with the manufacturer's protocol, each supernatant sample was measured in duplicates.

\section{ROS detection}

Intracellular ROS were determined by non-fluorescent probe 20, 70-dichlorofluorescin diacetate (DCFH-DA) in accordance with the manufacturer's protocol. Briefly, cells were digested by $0.25 \%$ trypsin-EDTA, followed by being treated with DCFH-DA $(10 \mathrm{mM})$ in the dark at $37^{\circ} \mathrm{C}$ for $30 \mathrm{~min}$, gently washed by PBS and observed under a fluorescence microscope. Cellular fluorescence intensity was determined in a microplate reader (RT-7300, Rayto, Guangdong, China) at an excitation of $485 \mathrm{~nm}$ and an emission of $538 \mathrm{~nm}$.

\section{MDA and total antioxidation capability (T-AOC) measurement}


Total cellular protein was extracted by cell lysis buffer supplemented with protease inhibitors and quantified via BCA assay. Afterwards, MDA concentration was measured by an MDA kit according to its instruction. Briefly, cellular protein samples were mixed with reagents provided in the kit and kept in a boiling water bath at $100^{\circ} \mathrm{C}$ for $15 \mathrm{~min}$. Then the mixtures were centrifuged and supernatants were measured at $532 \mathrm{~nm}$ with a microplate reader. MDA level was expressed as $\mathrm{nmol} / \mathrm{ml}$. T-AOC was determined via Rapid ABTS method using a T-AOC assay kit according to the enclosed guidelines. Briefly, cellular protein samples were mixed with required reagents and incubated at room temperature for $5 \mathrm{~min}$; then the mixtures were measured at $405 \mathrm{~nm}$ by a Varioskan Flash spectral scanning multimode reader. TAOC level was expressed as umol/mg.

\section{Quantitative real-time polymerase chain reaction (qRT-PCR) analysis}

Total RNA was extracted from cell pellets using trizol reagent based on the manufacturer's instructions. RNA concentration and purity were measured with a spectrophotometer prior to cDNA synthesis.

Afterwards, cDNA was synthesized by reverse transcription of $600 \mathrm{ng}$ total RNA according to the guideline of a PrimeScriptTM RT reagent kit. Finally, genes of interest were amplified in accordance with SYBRR premix Ex TaqTM reagent kit's instructions under an ABI PRISM 7500 sequence detection system (Applied Biosystems, USA). Relative expression of target genes was normalized to that of housekeeping gene GAPDH and calculated by the $2^{-\triangle \Delta C t}$ method. Primers used for qRT-PCR analysis were shown in Table 1 .

Table 1: Primers used for qRT-PCR analysis

\begin{tabular}{lll}
\hline Gene (human) & & Sequence $\left(5^{\prime}-3{ }^{\prime}\right)$ \\
\hline \multirow{2}{*}{ GAPDH } & Forward & TGCCACTCCTCCACCTTTG \\
& Reverse & CGAACCACCCTGTTGCTGT \\
\multirow{2}{*}{ NF-kB } & Forward & ACAACCCCTTCCAAGTTCCT \\
& Reverse & TGGTCCCGTGAAATACACCT \\
Nrf2 & Forward & AAACCAGTGGATCTGCCAAC \\
& Reverse & ACGTAGCCGAAGAAACCTCA \\
NOX1 & Forward & CTGGGTGGTTAACCACTGGTT \\
& Reverse & ACCAATGCCGTGAATCCCTAAG \\
\hline
\end{tabular}

\section{Western blot analysis}

Whole cell protein extractions and quantifications were performed as described above. Subsequently, cell lysates with an equal amount of total protein $(40 \mu \mathrm{g})$ were separated by sodium dodecyl sulfatepolyacrylamide gel electrophoresis and then electrophoretically transferred to polyvinylidene difluoride membranes. The membranes were blocked with $5 \%$ skimmed milk for four hours, and then were incubated with rabbit anti-human primary antibodies of GAPDH (1:5000), Nrf2 (1:2000), NF-KB p65 (1:1000), and NOX1 (1:1000) overnight at $4^{\circ} \mathrm{C}$. Afterwards, the membranes were washed and incubated 
with goat anti-rabbit horseradish peroxidase-conjugated secondary antibody (1:6000) for one hour. Lastly, proteins of interest were visualized via an ECL detection reagent by an ImageQuant LAS4000 chemiluminescence detection system. All images were analyzed using the Quantity One software. GAPDH served as a loading control and expressions of target proteins were normalized.

\section{Luciferase activity assay}

AliBaba2.1 database (http://gene-regulation.com/pub/programs/alibaba2/) was applied to predict potential transcription factor binding site in the promoter of NOX1. Subsequently, the fragment in 5'flanking region of NOX1 promoter (1500bp) and that of deletion mutant (1489bp), of which the predicted binding site was deleted were cloned, amplified and inserted into pGL3 basic plasmid to construct recombinant plasmids, namely "pGL3-NOX1-1500" plasmid and "pGL3-NOX1-1489" plasmid. After being transfected with these plasmids, cells were stimulated by TNF- $a$ and then luciferase activity was monitored on a MD SpectraMax M5 enzyme-labeled instrument and calculated as a ratio of firefly luciferase relative to Renilla luciferase luminescence.

\section{Statistical analysis}

Statistical analyses were performed using SPSS21.0 (IBM Analytics, Armonk, NY, USA). Data were represented as means \pm standard deviation, and analyzed by independent-samples t-test and one-way ANOVA. $P$ values $<0.05$ was considered to be statistically significant.

\section{Results}

\section{TNF- $\mathrm{a}$ upregulated NOX1 and Nrf2 expressions in $\mathrm{T}_{2} \mathrm{AECs}$}

To examine the regulatory effects of TNF-a on the expression of NOX1 and Nrf2 in $\mathrm{T}_{2} \mathrm{AECs}$, the expression profiles of NOX1 and Nrf2 in A549 cells following TNF-a induction were measured. As shown in Figure 1, the expressions of NOX1 and Nrf2, both in mRNA and protein levels, were apparently increased with the increasing concentration of TNF- $a$, reaching their maximum at $10 \mathrm{ng} / \mathrm{ml}$, simultaneously $(P<0.05)$. There were no statistical differences among 10,20 and $40 \mathrm{ng} / \mathrm{ml}$ groups (Pख0.05). Similarly, TNF-a significantly upregulated the levels of NOX1 and Nrf2 as the incubation time was prolonged, exerting its maximum effects at 24 hours $(P<0.05)$. There were no statistical differences among 24,36 and 48 hours groups (P凶0.05).

\section{The effects of different vectors on Nrf2 expression induced by TNF- $a$ in $T_{2} A E C s$}

To test the effects of different vectors on TNF-a-mediated expression of Nrf2 in $\mathrm{T}_{2} \mathrm{AECs}$, mRNA and protein expressions of Nrf2 were measured in A549 cells in different groups. As shown in Figure 2, compared with naive group, the expressions of Nrf2 were decreased in Nrf2 siRNA group while were increased in TNF-a-treated group $(P<0.05)$, negative control group $(P<0.05)$ and empty vector group $(P<0.05)$. Notably, the levels of Nrf2 were further increased in Nrf2 overexpression vector group $(P<0.05)$. 
There were no statistical differences among TNF-a-treated group, negative control group and empty vector group $(P \otimes 0.05)$.

\section{Nrf2 reduced NF-KB expression induced by TNF- $\mathrm{a}$ in $\mathrm{T}_{2} \mathrm{AECs}$}

To investigate the effect of Nrf2 on regulating TNF-a-mediated expression of NF-kB in $\mathrm{T}_{2} \mathrm{AECs}$, mRNA and protein expressions of NF-KB were measured in A549 cells. As shown in Figure 2, compare with naïve group, the expressions of NF-KB in TNF-a-treated group $(P<0.05)$, negative control group $(P<0.05)$ and empty vector group $(P<0.05)$ were significantly increased, and the highest level was observed in Nrf2 siRNA group $(P<0.05)$. Of note, NF-KB level in Nrf2 overexpression group was higher than that in naïve group $(P<0.05)$ while was lower than that in TNF-a-treated group $(P<0.05)$. There were no statistical differences in terms of NF-KB level among TNF-a-treated group, negative control group and empty vector group ( $P \otimes 0.05)$.

\section{Nrf2 reduced IL-6, IL8, ROS and MDA productions induced by TNF- $a$ in $T_{2} A E C s$ and increased T-AOC of $\mathrm{T}_{2} \mathrm{AECs}$ under TNF- $\mathrm{a}$ challenge}

To assess the regulatory effects of Nrf2 on inflammation reaction and oxidative stress damage in $\mathrm{T}_{2} \mathrm{AECs}$ induced by TNF-a, levels of IL-6, IL8, ROS, MDA, and T-AOC were measured in A549 cells. As shown in Figure 3, compared with naive group, supernatant levels of IL-6 and IL-8 in TNF-a-treated group $(P<0.05)$, negative control group $(P<0.05)$ and empty vector group $(P<0.05)$ were obviously increased, and those in Nrf2 siRNA group were further elevated $(P<0.05)$. Of note, the expressions of IL-6 and IL8 in Nrf2 overexpression group were lower than those in TNF-a-treated group $(P<0.05)$ while were higher than those in naïve group $(P<0.05)$. Levels of ROS $(P<0.05)$ and MDA $(P<0.05)$ shared similar trends with those of IL6 and IL-8. In contrast, compared with naïve group, T-AOC level in TNF-a-treated group $(P<0.05)$, negative control group $(P<0.05)$ and empty vector group $(P<0.05)$ were decreased, reaching its lowest in Nrf2 siRNA group. Notably, T-AOC level in Nrf2 overexpression group was lower than that in naïve group $(P<0.05)$ whilst was higher than that in TNF-a-treated group $(P<0.05)$. There were no statistical differences with regards to IL-6, IL8, ROS, MDA, and T-AOC levels among TNF-a-treated group, negative control group and empty vector group ( $P \otimes 0.05)$.

\section{Nrf2 inhibited NOX1 expression induced by TNF- $a$ in $T_{2} A E C s$ through binding to Nrf2 binding element in NOX1 proximal promoter}

To verify whether Nrf2 is able to regulate TNF- $a$ induced NOX1 expression in $\mathrm{T}_{2} \mathrm{AECs}$, NOX1 expression profiles were determined in A549 cells subsequence to aforementioned manipulations. As shown in Figure 4, expressions of NOX1, both in mRNA and protein levels, were dramatically increased by TNF-a $(P<0.05)$, reaching their peaks in Nrf2 siRNA group $(P<0.05)$ while those in Nrf2 overexpression group were notably declined $(P<0.05)$. There were no statistical differences in terms of the expression of NOX1 among TNF- $a$ treated group, negative control group and empty vector group ( $P \otimes 0.05)$. To further study the potential mechanism by which Nrf2 possesses the ability to inhibit the expression of NOX1, the effect of 
Nrf2 binding element deletion on translational activity of NOX1 promoter in A549 cells were investigated. As shown in Figure 5, the sequence of NOX1 promoter cloned in this study was in line with the official sequence documented in the NCBI website, and the only difference between the deletion mutant and the official one is that the predicted Nrf2 binding element sequence was absent in the deletion mutant. Agarose gel electrophoresis showed that the recombinant plasmid named "pGL3-NOX1-1500" was the combination of pGL3 plasmid andNOX1 promoter in full-length, and that named "pGL3-NOX1-1489" was the combination of pGL3 basic plasmid and the deletion mutant. Cells in "pGL3-NOX1-1500"group had the highest luciferase activity, followed by those in "pGL3-NOX1-1489" group and pGL3 group $(P<0.05)$.

\section{Discussion}

ALI has been posing great burden and challenge to humans in terms of health and economy ${ }^{[2]}$, highlighting an urgent need of a better understanding of its pathogenesis. Fierce inflammatory reaction and imbalanced oxidative stress in $\mathrm{T}_{2} \mathrm{AECs}$ are the major contributors to the pathogenesis and development of $A L I{ }^{[6,17]}$. NOX1 and Nrf2 are two pivotal mediators with regards to the maintenance of inflammatory reaction and oxidative stress levels, from this perspective, it is worthy to investigating the role of the crosstalk between these two factors in ALI pathogenesis.

In this study, TNF-a-induced A549 cells were used to explore the effects of the interaction between NOX1 and Nrf2 on the pathogenesis of ALI and the underlying mechanisms. Accumulating evidences showed that the concentrations of IL- 6 and IL-8, two presentative proinflammatory cytokines, were increased in $\mathrm{ALI}$ and their levels were positively correlated to the severity of pulmonary pathological abnormalities.

Thus, the concentrations of IL- 6 and IL- 8 were measured to assess the level of inflammation reaction $[2,18$, 19]. Owing to its wide application in assessing the level of oxidative stress, MDA was used as an oxidative stress level marker ${ }^{[20]}$. Of note, our results indicated that Nrf2 might be able to downregulate the expression of NOX1 by binding to the promoter of NOX1, by which Nrf2 significantly reduced the levels of IL-6, IL-8, ROS and MDA in $T_{2} A E C s$ while efficiently increased the T-OAC of $T_{2} A E C s$ under ALI. In addition, the expression of NF-KB was greatly inhibited by Nrf2, which might also contribute to the attenuation of oxidative damages caused by TNF-a.

The uncontrolled inflammatory response can cause abnormal production and accumulation of ROS in various structural cells, which in turn leads to the break of the balance between the oxidant and antioxidant defense systems and brings severe outcomes, including oxidative damages or even death to structural cells ${ }^{[21]}$. NOX family members are the predominant sources of ROS, and NOX1 is the main oxidase in regulating the level of ROS in lungs, playing significant roles in many oxidative stress associated pulmonary diseases, including ALI ${ }^{[8]}$. Previous studies showed that oxidative stress damages were observed in lungs in hypoxia-induced ALI mice, and wild type mice were more susceptible to developed severe pathological abnormalities, including increased lung permeability, inflammation infiltration, and pulmonary epithelial damage, than their $\mathrm{NOX} 1^{-/}$counterparts ${ }^{[22,23]}$. Yuan and colleagues illustrated that TNFR-Fc fusion protein reduced oxidative stress damage by inhibiting the level of 
NOX1/ROS in lungs in LPS-induced ALI ${ }^{[24]}$. Additionally, many research demonstrated that the expression of NOX1 was upregulated by TNF-a, leading to excessive oxidative stress injury to alveolar epithelial cells $[22,25]$. Our results were in line with these investigations, showing that TNF-a markedly upregulated the expression of NOX1 in concentration-independent and time-independent manners (the maximum level of NOX1 was seen when $T_{2}$ AECs was stimulated by $10 \mathrm{ng} / \mathrm{ml}$ TNF-a for 24 hours).

Nrf2 is a predominant regulator of cellular defense mechanism against oxidative stress because it can regulate the expression activity of various genes which are crucial in response to oxidative stress ${ }^{[10,11]}$. Recently, Nrf2 has been treated as a potent terminator for oxidative stress injury due to its ability in greatly reducing oxidative stress level in alveolar epithelial cells. Hence, it has become one master cytoprotective mediator for alveolar epithelia cells in $A{ }^{[}{ }^{[12,13]}$. However, the underlying mechanism by which Nrf2 exerts its protective effects in TNF-a-induced T2AECs is not fully elaborated. As confirmed in this study, the expressions of Nrf2 and NOX1 were simultaneously upregulated by TNF-a induction, furthermore, there might be an Nrf2 binding element in the promoter of NOX1 based on the prediction of AliBaba2.1 database, thus, it is of great value to explore whether Nrf2 is able to modulate the expression activity of NOX1, which in turn reduces the generations of ROS and proinflammatory cytokines, reconstructs the redox homeostasis and ultimately attenuates the oxidative stress damage induced by TNF- $a$ in $T_{2} A E C s$. As predicted, our study demonstrated that, under the stimulation of TNF-a, with the increasing expression of Nrf2, the expression activity of NOX1 in terms of transcription and translation was significantly reduced, consistently, the levels of IL-6, IL-8, ROS and MDA were decreased and T-OAC was increased. Conversely, reducing the expression of Nrf2 greatly enhanced the expression activity of NOX1, reduced the productions of IL-6, IL-8, ROS and MDA as well as raised the level of T-OAC. These finding firmly indicated that NOX1 plays a central role in disrupting the equilibrium between oxidant system and antioxidant system of $\mathrm{T}_{2} \mathrm{AEC}$ in $\mathrm{ALI}$ and Nrf2 exhibits its protective roles in ALI by downregulating the transcription and translation of NOX1. Thus, the suppression effect of Nrf2 on the expression of NOX1 greatly contributes to the reconstruction of redox hemostasis in T2AECs induced by TNF-a, making Nrf2 become a cellular pro-survival factor in ALI.

Nonetheless, the way that Nrf2 suppresses the expression of NOX1 is still undiscovered. Given that the Nrf2-antioxidant response element signaling strongly facilitates to maintain the balance between oxidant system and antioxidant system under oxidative stress condition ${ }^{[11]}$ and that the existence possibility of Nrf2 binding element in the promoter of NOX1, it is reasonable to assume that the predicted binding element plays a pivotal role in the interaction between Nrf2 and NOX1. To confirm this hypothesis, the sequence of Nrf2 binding element was deleted and a deletion mutant of NOX1 promoter were synthesized by overlap extension PCR, after that, two recombinant plasmids were constructed by inserting the sequence of full-length of NOX1 promoter and that of the deletion mutant to the pGL3 basic plasmid and transformed these plasmids into A549 cells under the induction of TNF-a. Luciferase activity results indicated that Nrf2 binding element allowed Nrf2 bind to the promoter of NOX1, by which Nrf2 can regulate the transcription activity of NOX1. 
Similarly, NF-KB also acts as a nuclear transcriptional regulator and is closed related to the pathogenesis of $\mathrm{ALI}{ }^{[26]}$. More importantly, NF-KB also possesses the ability to regulate the expression activity of NOX1, via binding its element in the promoter of $\mathrm{NOX} 1^{[27]}$ and it is mainly activated by TNF-a ${ }^{[28]}$. Additionally, increasing evidence indicated that there is a crosstalk between Nrf2 and NF-KB ${ }^{[29,30]}$. Thus, NF-KB might participate in the downregulation of Nrf2 on the expression of NOX1 and the level of oxidative stress in $\mathrm{T}_{2} \mathrm{AEC}$ s induced by TNF-a. The potential interplay between Nrf2 and NF-KB in $\mathrm{T}_{2} \mathrm{AEC}$ s under the stimulation of TNF-a were explored. Interestingly, TNF-a significantly upregulated the expression of NF-KB, while the expression of NF-kB was obviously inhibited by Nrf2.

Collectively, this present study showed that Nrf2 is able to downregulate the expression of NOX1, thereby decreasing the productions of IL-6, IL-8, ROS and MDA in T $2 A E C$, reinforcing $T_{2} A E C s^{\prime}$ T-OAC and ultimately attenuating the levels of inflammation reaction and oxidative stress under ALI either directly by binding to its binding element in the promoter of NOX1 or indirectly by inhibiting the expression of NF-KB. There are some limitations in our study, including the mechanism for the interaction between Nrf2 and NOX1 is not fully investigated, neither does the roles that NF-KB takes part in the regulation of Nrf2 on NOX1 expression. However, chromatin immunoprecipitation assay is in progress, which will definitely contribute to gain a clear picture of the interplay between these factors and better the understanding of the mechanism by which Nrf2 has the cytoprotective effect in ALI.

\section{Conclusion}

Taken together, Nrf2 plays essential roles in mitigating inflammation reaction and oxidative stress damage in $\mathrm{T}_{2} \mathrm{AEC}$ induced by TNF-a through inhibiting NOX1 signalling pathway. Hence, Nrf2 might be a promising target for ALI therapy.

\section{Abbreviations}

ALI: acute lung injury; $T_{2} A E C s$ : type II alveolar epithelial cells; IL: interleukin; ROS: reactive oxygen species; MDA: malondialdehyde; T-AOC: total antioxidation capability.

\section{Declarations}

\section{Ethics approval and consent to participate}

Not applicable.

\section{Consent for publication}

Not applicable.

\section{Availability of data and materials}


All data generated or analyzed during this study are included in this published article.

\section{Funding}

This research was sponsored by the National Natural Science Foundation of China (No. 81541002); Fujian Provincial Natural Science Foundation (2015J05147) and Science Launch Foundation of Fujian Medical University (2018QH1097).

\section{Competing interests}

The authors declare that they have no competing interests.

\section{Authors' contributions}

WJW, ZXZ and YMZ conceived the study. WJW, JMZ, XPL, XSS, LZ and ZSZ analyzed the data. WJW, $J M Z$ and $X H L$ created the figures and tables. WJW, JMZ, XHL and ZXZ wrote the first draft. XHL, XSS, $X P L, L Z$ and ZSZ edited the paper. WJW, YMZ and ZXZ critically reviewed the paper. All authors discussed the results and contributed to the final manuscript.

\section{Authors' information}

${ }^{1}$ Department of Pulmonary and Critical Care Medicine, the Second Affiliated Hospital of Fujian Medical University, Respirology Medicine Centre of Fujian Province, 34 Zhongshanbei Road, Licheng District, Quanzhou, China; ${ }^{2}$ Department of Radiology, Fujian Medical University Affiliated First Quanzhou Hospital, 248-252 East Road, Licheng District, Quanzhou, China; ${ }^{3}$ Department of Ultrasound Medicine, the Second Affiliated Hospital of Fujian Medical University, 34 Zhongshanbei Road, Licheng District, Quanzhou, China; ${ }^{4}$ Department of Medical Imaging, Quanzhou Jinjiang Anhai Hospital, Haiba Middle Road, Anhai Town, Jinjiang, Quanzhou, China

\section{Acknowledgements}

Not applicable.

Conflict of interest statement: All the authors declare that there is no conflict of interest regarding the publication of this article.

\section{References}

1. Hayes, M., et al., Clinical review: Stem cell therapies for acute lung injury/acute respiratory distress syndrome - hope or hype? Crit Care, 2012. 16(2): p. 205.

2. Butt, Y., A. Kurdowska, and T.C. Allen, Acute Lung Injury: A Clinical and Molecular Review. Arch Pathol Lab Med, 2016. 140(4): p. 345-50. 
3. Mowery, N.T., W.T.H. Terzian, and A.C. Nelson, Acute lung injury. Curr Probl Surg, 2020. 57(5): p. 100777.

4. Zhu, Z., Lian, X., Su, X. et al. From SARS and MERS to COVID-19: a brief summary and comparison of severe acute respiratory infections caused by three highly pathogenic human coronaviruses. Respir Res 21, 224 (2020). https://doi.org/10.1186/s12931-020-01479-w.

5. Hughes, K.T. and M.B. Beasley, Pulmonary Manifestations of Acute Lung Injury: More Than Just Diffuse Alveolar Damage. Arch Pathol Lab Med, 2017. 141(7): p. 916-22.

6. Sarma, J.V. and P.A. Ward, Oxidants and redox signaling in acute lung injury. Compr Physiol, 2011. 1(3): p. 1365-81.

7. Sies, H. and D.P. Jones, Reactive oxygen species (ROS) as pleiotropic physiological signalling agents. Nat Rev Mol Cell Biol, 2020. 21(7): p. 363-83.

8. Schröder, K., NADPH oxidases: Current aspects and tools. Redox Biol, 2020. 34: p. 101512.

9. Pendyala, S. and V. Natarajan, Redox regulation of Nox proteins. Respir Physiol Neurobiol, 2010. 174(3): p. 265-71.

10. Mou, Y., et al., Recent progress in Keap1-Nrf2 protein-protein interaction inhibitors. Eur J Med Chem, 2020. 202: p. 112532.

11. Shaw, P. and A. Chattopadhyay, Nrf2-ARE signaling in cellular protection: Mechanism of action and the regulatory mechanisms. J Cell Physiol, 2020. 235(4): p. 3119-30.

12. Zhang, S., et al., Nrf2 transfection enhances the efficacy of human amniotic mesenchymal stem cells to repair lung injury induced by lipopolysaccharide. J Cell Biochem, 2018. 119(2): p. 1627-36.

13. Liu, Q., Y. Gao, and X. Ci, Role of Nrf2 and Its Activators in Respiratory Diseases. Oxid Med Cell Longev, 2019. 2019: p. 7090534.

14. Malaviya, R., J.D. Laskin, and D.L. Laskin, Anti-TNFa therapy in inflammatory lung diseases. Pharmacol Ther, 2017. 180: p. 90-8.

15. Mukhopadhyay, S., J.R. Hoidal, and T.K. Mukherjee, Role of TNFalpha in pulmonary pathophysiology. Respir Res, 2006. 7(1): p. 125.

16. Yang, H.M., et al., Pogostone attenuates TNF-a-induced injury in A549 cells via inhibiting NF-KB and activating Nrf2 pathways. Int Immunopharmacol, 2018. 62: p. 15-22.

17. Kellner, M., et al., ROS Signaling in the Pathogenesis of Acute Lung Injury (ALI) and Acute Respiratory Distress Syndrome (ARDS). Adv Exp Med Biol, 2017. 967: p. 105-37.

18. Allen, T.C. and A. Kurdowska, Interleukin 8 and acute lung injury. Arch Pathol Lab Med, 2014. 138(2): p. 266-9.

19. Zhu, Z., et al., Point-of-Care Ultrasound-A New Option for Early Quantitative Assessment of Pulmonary Edema. Ultrasound Med Biol, 2020. 46(1): p. 1-10.

20. Mittal, M., et al., Reactive oxygen species in inflammation and tissue injury. Antioxid Redox Signal, 2014. 20(7): p. 1126-67. 
21. Su, L.J., et al., Reactive Oxygen Species-Induced Lipid Peroxidation in Apoptosis, Autophagy, and Ferroptosis. Oxid Med Cell Longev, 2019. 2019: p. 5080843.

22. Carnesecchi, S., et al., NADPH oxidase-1 plays a crucial role in hyperoxia-induced acute lung injury in mice. Am J Respir Crit Care Med, 2009. 180(10): p. 972-81.

23. Carnesecchi, S., et al., NOX1 is responsible for cell death through STAT3 activation in hyperoxia and is associated with the pathogenesis of acute respiratory distress syndrome. Int $\mathrm{J}$ Clin Exp Pathol, 2014. 7(2): p. 537-51.

24. Yuan, W.F., et al., [TNFR-Fc prevents lipopolysaccharide-induced acute lung injury in mice via oxidative stress inhibition]. Zhonghua Jie He He Hu Xi Za Zhi, 2012. 35(6): p. 435-9.

25. Oshima, H., et al., TNF-a/TNFR1 signaling promotes gastric tumorigenesis through induction of Noxo1 and Gna14 in tumor cells. Oncogene, 2014. 33(29): p. 3820-9.

26. Hu, R., et al., The role of TLR4 in the pathogenesis of indirect acute lung injury. Front Biosci (Landmark Ed), 2013. 18: p. 1244-55.

27. Morgan, M.J. and Z.G. Liu, Crosstalk of reactive oxygen species and NF-KB signaling. Cell Res, 2011. 21(1): p. 103-15.

28. Liu, Y., et al., TRIM25 Promotes TNF-a-Induced NF-kB Activation through Potentiating the K63-Linked Ubiquitination of TRAF2. J Immunol, 2020. 204(6): p. 1499-507.

29. de Almeida, A., et al., Unveiling the Role of Inflammation and Oxidative Stress on Age-Related Cardiovascular Diseases. Oxid Med Cell Longev, 2020. 2020: p. 1954398.

30. Wardyn, J.D., A.H. Ponsford, and C.M. Sanderson, Dissecting molecular cross-talk between Nrf2 and NF-kB response pathways. Biochem Soc Trans, 2015. 43(4): p. 621-6.

\section{Figures}



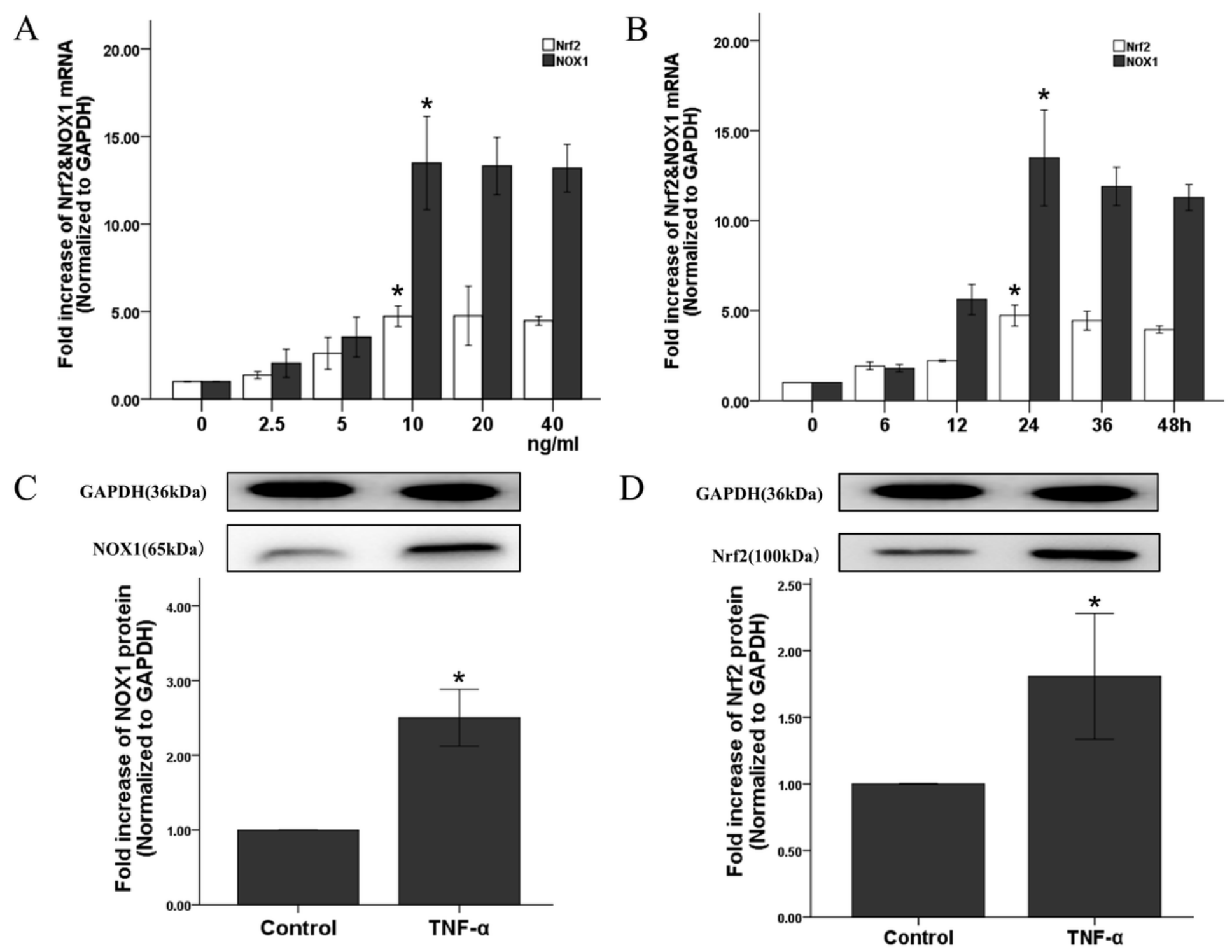

\section{Figure 1}

The effects of TNF- $a$ on Nrf2 and NOX1 expression in T2AECs. A. Nrf2 and NOX1 mRNA induced by TNF$a$ at different concentrations. B. Nrf2 and NOX1 mRNA induced by TNF-a in time-dependent manners. C. NOX1 protein induced by $10 \mathrm{ng} / \mathrm{ml}$ TNF-a for 24 hours. D. Nrf2 protein induced by $10 \mathrm{ng} / \mathrm{ml}$ TNF-a for 24 hours. ${ }^{*}<0.05$, compared with the control group. 


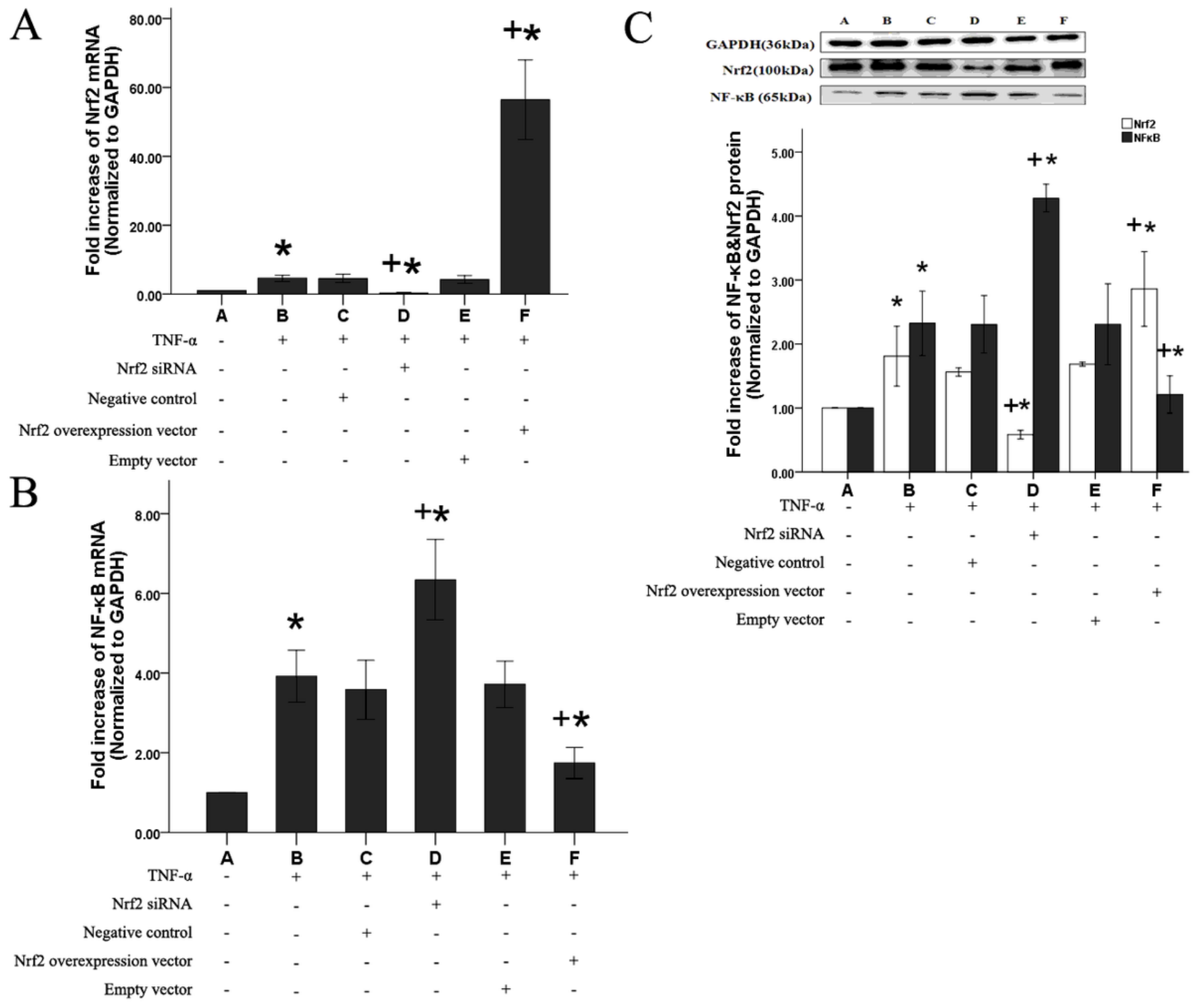

Figure 2

Nrf2 mRNA expression profiles and the effects of Nrf2 on NF-kB expression. A. The expression level of Nrf2 mRNA in different groups. B. The effects of Nrf2 on the transcription of NF-kB (mRNA). C. The effects of Nrf2 on the translation of NF-KB (protein). ${ }^{*} \mathrm{P}<0.05$, compared with the control group. $+\mathrm{P}<0.05$, compared with the TNF-a-treated group. 


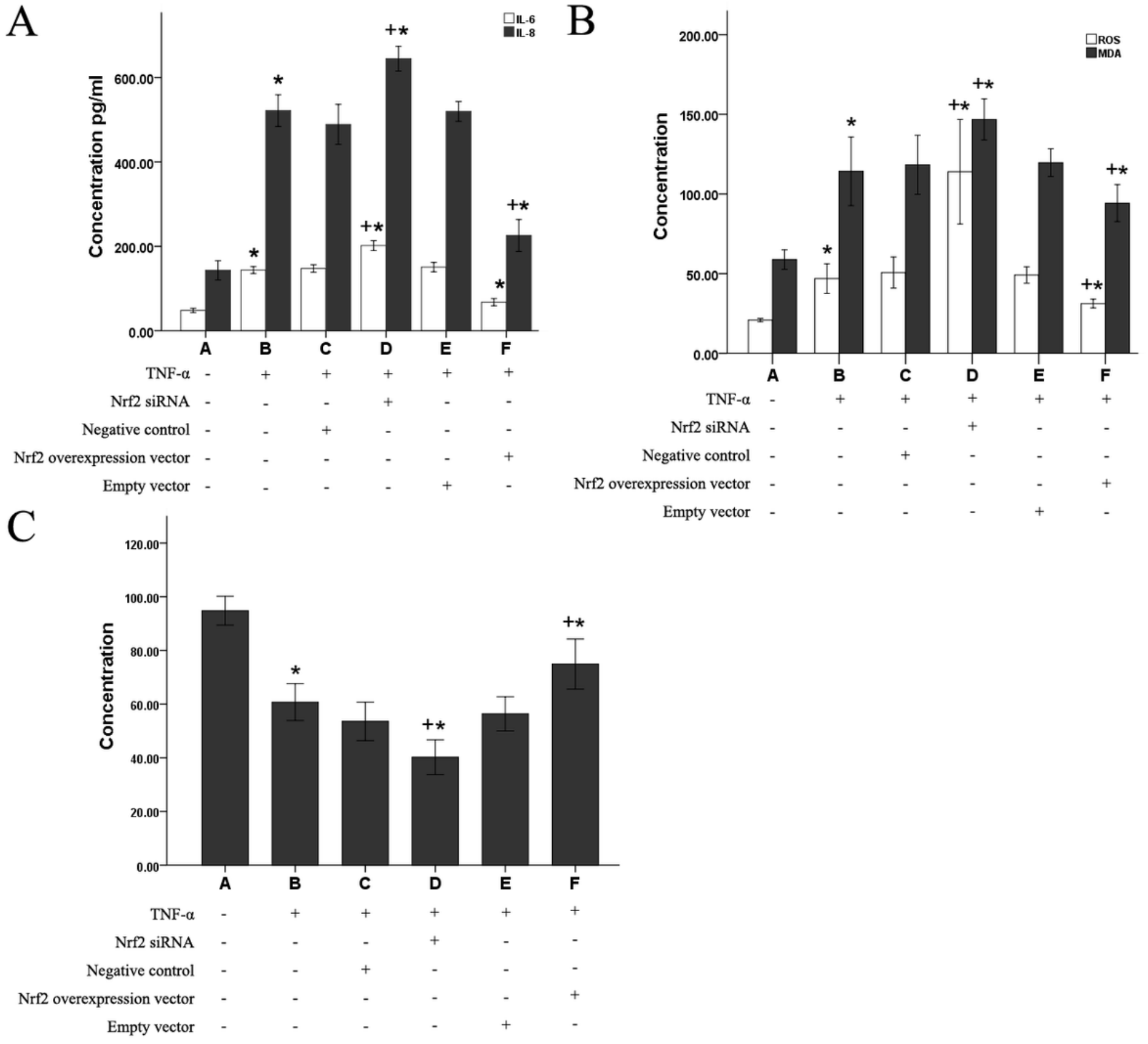

\section{Figure 3}

The effects of Nrf2 on the level of inflammatory reaction and oxidative stress. A. The effects of Nrf2 on the productions of IL- 6 and IL-8. B. The effects of Nrf2 on the productions of ROS and MDA. C. The effects of Nrf2 on the T-AOC level. ${ }^{*} P<0.05$, compared with the control group. $+P<0.05$, compared with the TNF-a-treated group. 

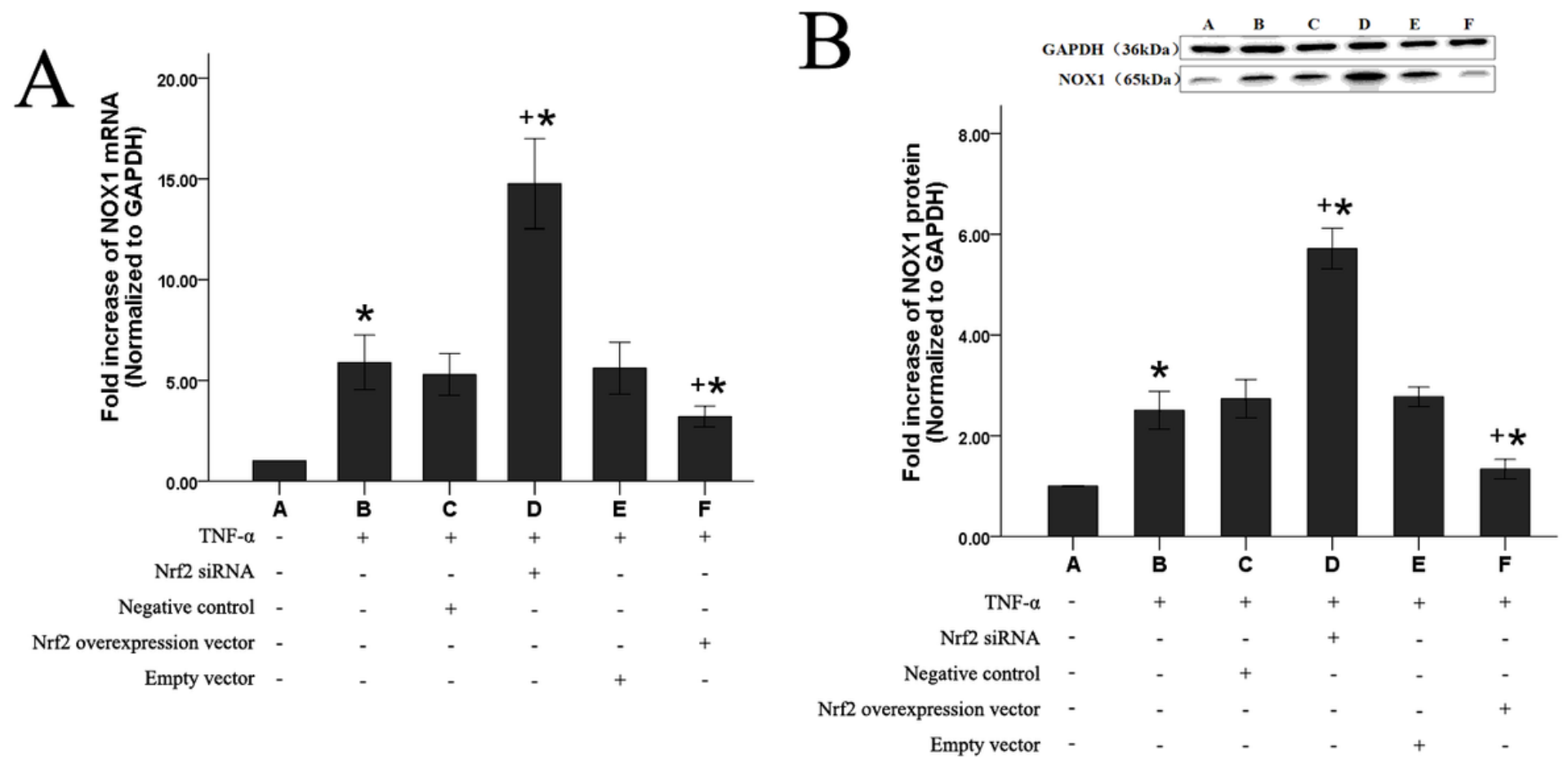

Figure 4

The effects of Nrf2 on the expression of NOX1. A. The effects of Nrf2 on the transcription of NOX1 (mRNA). B. The effects of Nrf2 on the translation of NOX1 (protein). ${ }^{*} \mathrm{P}<0.05$, compared with the control group. $+\mathrm{P}<0.05$, compared with the TNF-a-treated group. 
A

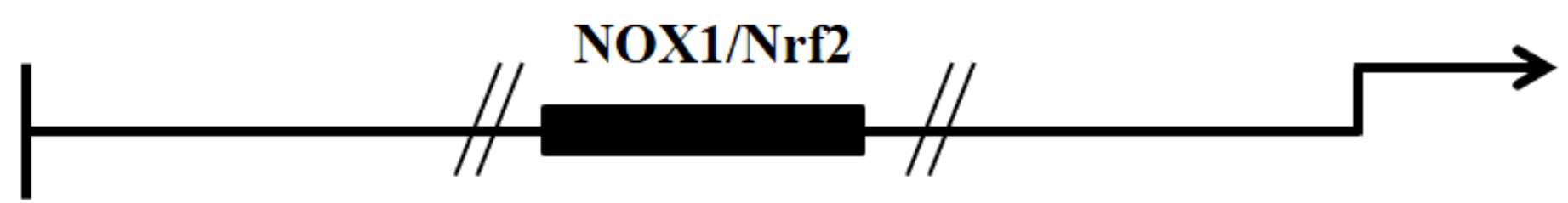

$-1500$

attacacagca

$+1$

$(-1199 \sim-1189)$

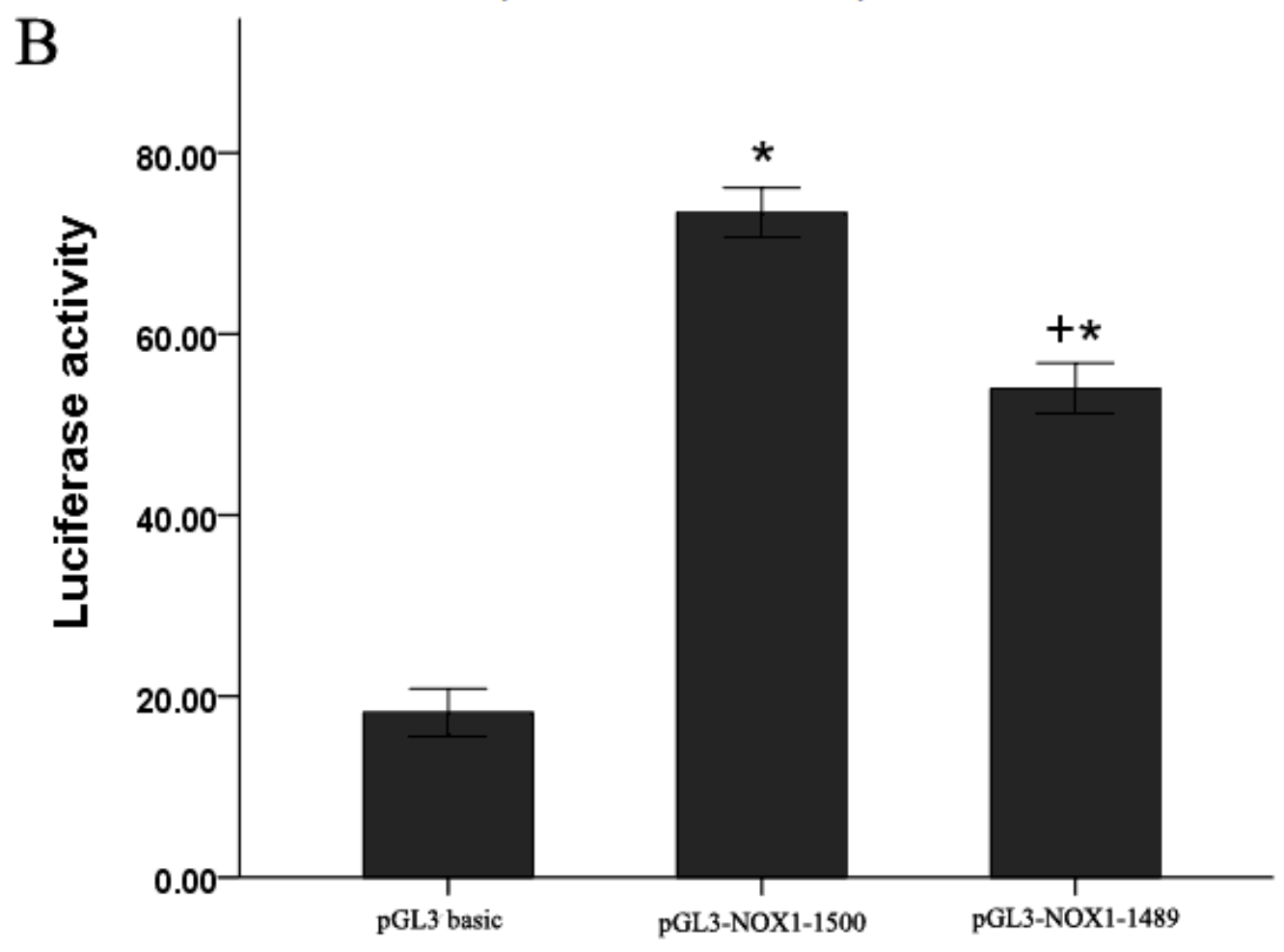

Figure 5

The effect of the Nrf2 binding element on the activity of NOX1 promoter. A. Nrf2 binding element in the promoter of NOX1, the location of the binding sequence was counted relative to the ATG codon; B.

Luciferase activity in different groups. ${ }^{*} P<0.05$, compared with the pGL3 basic group. $+P<0.05$, compared with the pGL3-NOX1-1500 group. 